Review Article

\title{
Rehabilitation and early mobilization in the critical patient: systematic review
}

\author{
Patricia Arias-Fernández, RN'), Macarena Romero-Martin, RN, MHS ${ }^{2}$, \\ Juan Gómez-Salgado, $\mathrm{PhD}^{3,4)^{*}}$, Daniel Fernández-García, $\mathrm{PhD}^{5 \text { ) }}$ \\ 1) Health Sciences School, Department of Nursing and Physiotherapy, Intensive Care Unit, \\ University Hospital of León, Spain \\ 2) Red Cross Nursing School, University of Sevilla, Spain \\ 3) Nursing School, University of Huelva: 21071 Huelva, Spain \\ 4) University Espiritu Santo, Ecuador \\ 5) Health Sciences School, Department of Nursing and Physiotherapy, University of León, Spain
}

\begin{abstract}
Purpose] To review the literature that examines rehabilitation and early mobilization and that involves different practices (effects of interventions) for the critically ill patient. [Materials and Methods] A PRISMA-Systematic review has been conducted based on different data sources: Biblioteca Virtual en Salud, CINHAL, Pubmed, Scopus, and Web of Science were used to identify randomized controlled trials, crossover trials, and case-control studies. [Results] Eleven studies were included. Early rehabilitation had no significant effect on the length of stay and number of cases of Intensive Care Unit Acquired Weaknesses. However, early rehabilitation had a significant effect on the functional status, muscle strength, mechanical ventilation duration, walking ability at discharge, and health quality of life. [Conclusion] Rehabilitation and early mobilization are associated with an increased probability of walking more distance at discharge. Early rehabilitation is associated with an increase in functional capacity and muscle strength, an improvement in walking distance and better perception of the health-related quality of life. Cycloergometer and electrical stimulation can be used to maintain muscle strength. Further research is needed to establish stronger evidences.

Key words: Critical care, Early mobilization, Rehabilitation
\end{abstract}

(This article was submitted Apr. 3, 2018, and was accepted Jun. 29, 2018)

\section{INTRODUCTION}

Despite the technological advances in intensive medicine, a large number of patients who survive a critical illness have their quality of life decreased ${ }^{1,2)}$. This fact is associated with a multifactorial morbidity that can cause functional, physical, cognitive and/or psychological disabilities ${ }^{2,3)}$ which persist even over 5 years after discharge ${ }^{4-6)}$. In the critical patient management, interventions that promote long periods of immobilization are usually performed such as the use of mechanical ventilation, administration of drugs, sedatives, analgesics, drugs to control anxiety and agitation, etc ${ }^{7-9)}$. Weakness is a common complication and is associated with a severe disability and a long rehabilitation. In this line, the intensive care unit acquired weakness (ICUAW) is associated with joint contractures, thromboembolism, resistance to insulin, microvascular alterations, pressure ulcers, atelectasis, pneumonia, extension of the weaning period, delirium, increase in the days of income, increased mortality, and development of disabilities ${ }^{10-12}$. Rehabilitation and early mobilization are considered therapeutic strategies to prevent the development of ICUAW ${ }^{13)}$.

The concept of mobilization is large, complex, and interdisciplinary. It is energy consuming and consists of physical and

*Corresponding author. Juan Gómez-Salgado (E-mail: jgsalgad@gmail.com)

(C2018 The Society of Physical Therapy Science. Published by IPEC Inc.

(c) (1) $\odot$ This is an open-access article distributed under the terms of the Creative Commons Attribution Non-Commercial No Derivatives cc) 
psychological aspects ${ }^{14)}$, including activities that produce movement such as "active limbs exercising, actively moving or rolling in bed, sitting on the edge of the bed, etc." ${ }^{15}$. Mobilization is globally defined as "the physical activity that, performed with the appropriate intensity, produces physiological benefits for the organism", acting on the circulation, central and peripheral perfusion, ventilation, or state of consciousness ${ }^{16)}$. The term "early" refers to activities that are carried out from the initial physiological stabilisation and that continue during the ICU (Intensive Care Unit) stay ${ }^{17}$.

Early mobilization of patients is safe, feasible, and has positive results in ICU patients. However, it is not a common practice extended to all units ${ }^{17,18}$. The literature includes various mobilization therapies and previous systematic reviews. Three of them study the effects of early rehabilitation on patients undergoing mechanical ventilation ${ }^{19-21)}$; another one focuses on interventions aimed at preventing the ICUAW ${ }^{22}$; also, the one that examines interventions to improve physical capacity in patients who survive their disease at the $\mathrm{ICU}^{23}$. To analyse the effects of early mobilization in critically-ill patients is of vital importance, so the aim of this systematic review has been to review the literature that examines rehabilitation and early mobilization and that involves different practices (effects of interventions) in critically-ill patients.

\section{MATERIALS AND METHODS}

Methodologically the PRISMA declaration (Preferred Reporting Items for Systematic Reviews and Meta-Analyses) ${ }^{24)}$ was used to carry out the study. The search was carried out in the following online databases: Biblioteca Virtual en Salud (BVS), CINHAL, Pubmed, Scopus and Web of Science (WOS). In each of them, combinations of the following Descriptores en Ciencias de la Salud (Health Sciences Descriptors; DeCS, for its acronym in Spanish), and Medical Subject Headings (MeSH) descriptors were used: "critical care", "rehabilitation", "early mobilization". The descriptors were combined using the Boolean operator "AND". An example of the search strategy in the CINHAL database was "critical care" and "rehabilitation" and "early mobilization" that returned 14 results. The search was limited to published works from 2006 to 2016.

References of systematic reviews were analyzed to include studies of interest. The selection was made by two reviewers. The following inclusion criteria were followed:

- The population was to be formed by adults over the age of 18 admitted to anintensive care unit for at least 48 hours.

- Interventions had to be based on mobilization or early rehabilitation.

- Studies should correspond to randomized clinical trial, crossover trial, or case-control.

- The studies chosen for the analysis should be available in full text for the author, in English or in Spanish.

- The studies had to be published between 1 January 2006 and 29 May 2016.

The exclusion criteria included studies in which:

- A review of articles was included.

- The intervention began at discharge from the ICU.

- The intervention had begun at home prior to admittance.

- Programs or protocols specifically designed for quality improvement projects were described.

The methodological quality of the included studies was assessed using the PEDro scale ${ }^{25}$. This scale consists of the following items: choice criteria, random assignment, concealed allocation, comparable groups, blinded participant, blinded therapists, blinded evaluators, adequate follow-up (measures of at least one of the key results obtained from more than $85 \%$ of participans), intention to treat analyses, groups' comparison, ad hoc measures, and variability. Each criterion is scored as present or not present $(\mathrm{Yes}=1, \mathrm{No}=0)$, and a maximum score of 10 was established. All criteria score 1 except for the first one, which is unscored.

The ethical considerations are considered at the declaration's section at the end of the manuscript.

\section{RESULTS}

Following the search strategy, 178 results were obtained (44 in the Biblioteca Virtual en Salud, 15 in CINHAL, 59 in Pubmed, 21 in Scopus, 31 in Web of Science, and 8 in additional sources). Of them, 68 were discarded for being duplicated. After the revision of titles and summaries, 98 articles were excluded according to the exclusion criteria. Of the 12 full texts to deduct eligibility, one was excluded for failing to meet the inclusion criteria ${ }^{26}$. Finally, 11 articles were chosen for this review $^{9,27-36)}$. Figure 1 shows the flowchart of the selected studies.

Information on the results obtained by the authors was selected from each study. The main characteristics of the studies are collected in Table 1 .

The eligibility criteria for the selected sample were described in all studies. In two studies, there was no random allocation due to the type of case-control design ${ }^{35,36}$. In six studies, the allocation of the participants to the groups was carried out in a concealed way $9,27,29,31,32,34)$. The basic characteristics of the individuals were compared in four studies ${ }^{9,28,31,32)}$. The participants were blinded in two studies ${ }^{31,32)}$. In none of the studies, the therapists performing the interventions were blinded. The evaluators were blinded in five studies $9,29,31,33,34)$. The analysis was carried out by intention to treat in four studies $9,29,31,34)$. There are only three studies that can be considered of high methodological quality for meeting the criteria of concealed assignment, blinded evaluators, and intention-to-treat analysis ${ }^{9,31,34)}$.

A total of 913 individuals participated in the studies at the time of randomization or assignment, of which 415 (45.45\%) participants belonged to the intervention group and $435(47.64 \%)$ to the control group. Of the total assigned individuals, 


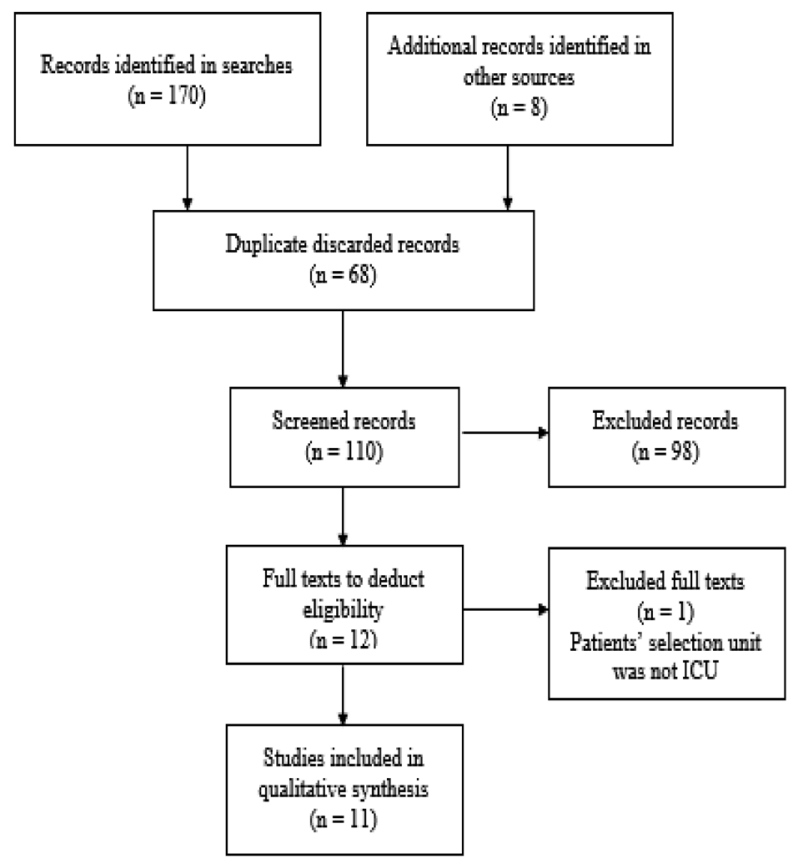

Fig. 1. Study selection flowchart.

347 (40.82\%) were women. The age and the APACHE II (Acute Physiology and Chronic Health Evaluation) score varied between the studies. The demographic characteristics of the participants are detailed in Table 2. Studies were conducted in medical $^{9,27,32,34,35)}$, surgical ${ }^{27,32,34,35)}$, and general-multivalent ICUs ${ }^{28-31,33,36)}$ and, in some cases, patients with critical ${ }^{35)}$ and coronary trauma ${ }^{34)}$ were included. The investigations were conducted in Australia ${ }^{29,31,34)}$, Belgium ${ }^{27)}$, Brazil ${ }^{28)}$, United States $^{9,32,33,35)}$ Japan $^{36)}$, New Zealand ${ }^{34)}$, and the United Kingdom ${ }^{30)}$.

The description of the interventions and the usual care applied in the control group, as well as the inclusion criteria of the participants, are reflected in Table 1. In general, therapies for progressive mobilization of passive and/or active exercises were applied to achieve ambulation. An intervention with cycloergometer was performed in three studies ${ }^{27-29)}$, and in two of them, electrostimulation was applied ${ }^{31,32)}$. Occupational therapy was part of the intervention in one study ${ }^{9)}$ and specific respiratory physiotherapy was applied in two investigations ${ }^{33,35)}$.

The frequency of early mobilization was daily $9,28,29,31-33,36)$ or 5 times a week ${ }^{27,35)}$. The frequency of usual care was performed daily, 5 , or 3 times a week, or was not recorded. The description of the interventions with the available data is shown in Table 3.

The start time of the intervention was collected in nine studies and varied from $1^{33)}$ to 14 days ${ }^{27)}$.

Then, the effects of interventions in the different studies related to functional capacity, muscle strength, mobility, quality of life, duration of mechanical ventilation, incidence of ICUAW, length of stay, and destination at discharge are described.

The functional capacity was measured by different instruments. Only two studies proved an effective intervention in improving functional capacities. One of them, at discharge from $\mathrm{ICU}^{35}$ ). The other one, at discharge from hospital ${ }^{9)}$.

In two studies, the intervention was effective in achieving an increase in muscle strength at discharge form the ICU ${ }^{27,}$ 28).

Mobility was assessed by a test or by taking into account the maximum distance walked with or without help. The intervention was effective at discharge from the hospital in three studies in a significant way ${ }^{9,27,32)}$.

The perceived quality of life was measured in three studies using the physical function item of the health questionnaire SF-36 (Health Survey questionnaire short form 36 Physical Functioning item). The evaluation was carried out at different times: at discharge from hospital ${ }^{27)}, 3$ months after discharge ${ }^{29}$, and 6 months after discharge ${ }^{31)}$. In the study by Burtin et al. ${ }^{27)}$, the quality of life was improved at discharge from hospital in a statistically significant manner.

The duration of mechanical ventilation was lower in the intervention group in a statistically significant way in two studies ${ }^{9,36)}$.

The proportion of ICUAW cases was collected in three studies ${ }^{9,29,34)}$. The differences were not statistically significant among the groups.

\section{DISCUSSION}

In this review, 11 studies have been analysed by evaluating interventions based on rehabilitation and early mobilization in the ICU, with a total of 850 participants assigned to the intervention and control groups, or to habitual care groups. 


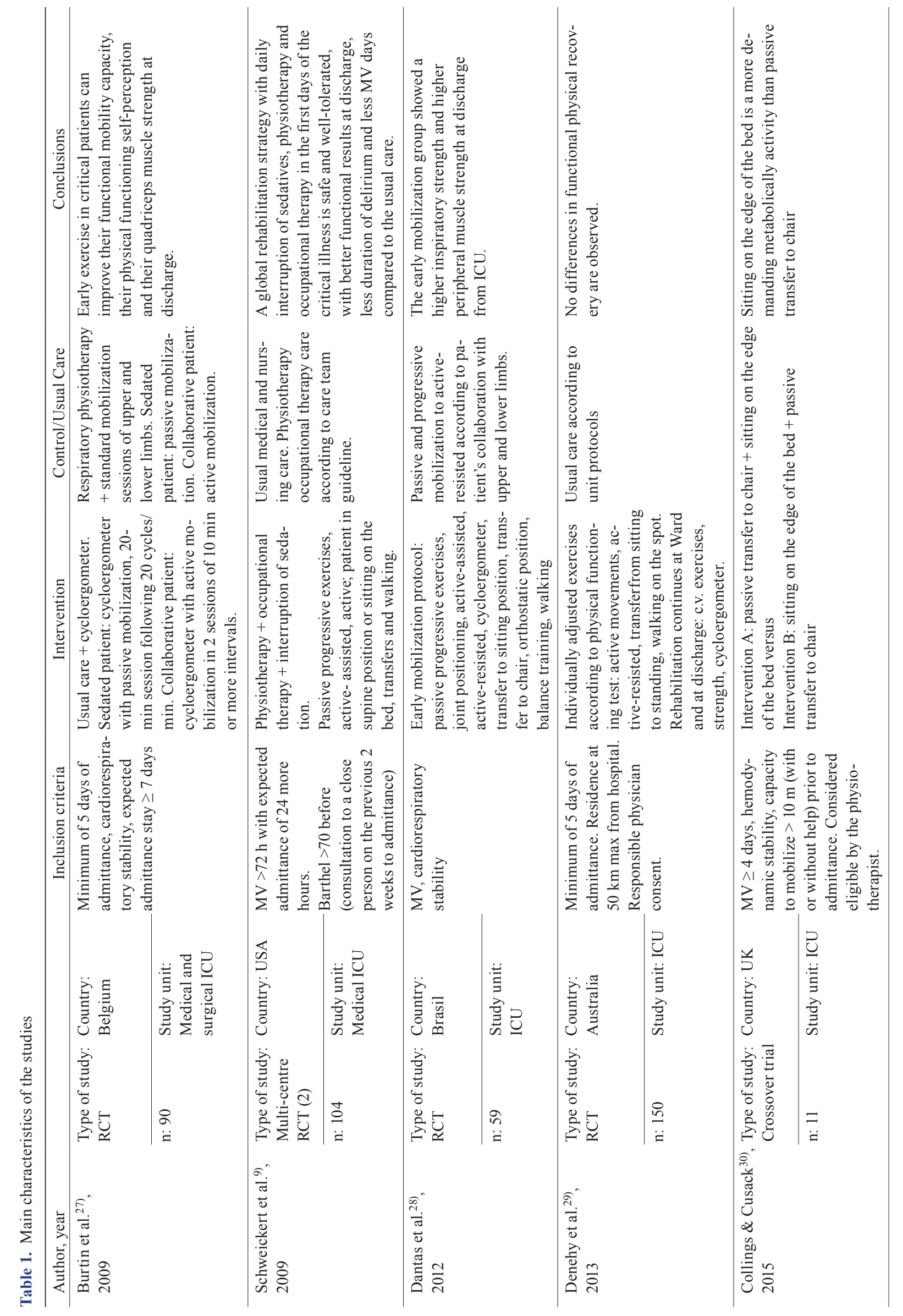




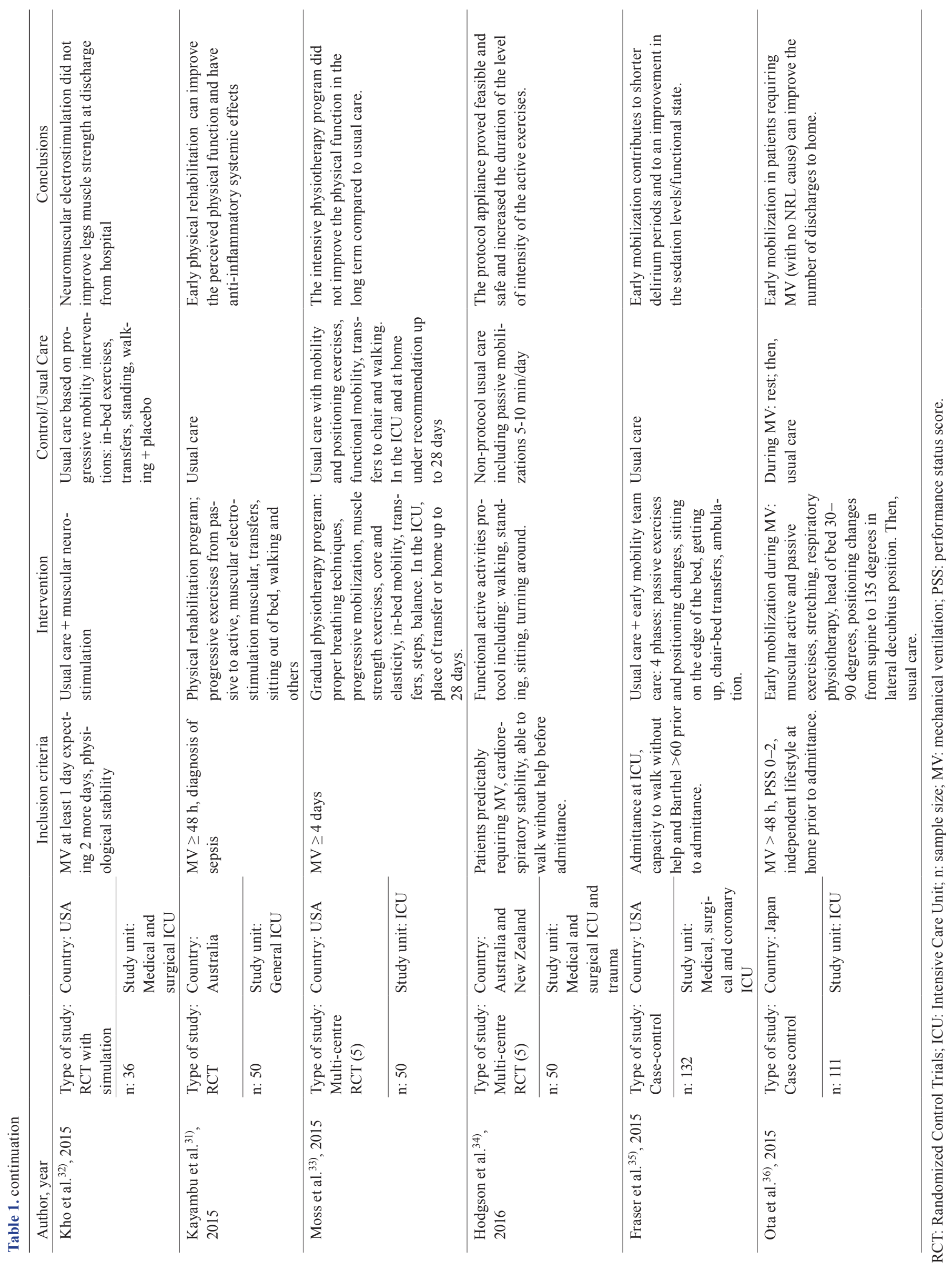


Table 2. Demographic characteristics of the participants

\begin{tabular}{|c|c|c|c|c|c|}
\hline Author, year & group & $\mathrm{n}$ & $\begin{array}{l}\text { Age }(\text { mean } \pm \text { SD or median } \\
\left.(\mathrm{IQR}) \text { or }(\mathrm{CI})^{*}\right)\end{array}$ & $\begin{array}{c}\text { Gender, } \mathrm{n}(\%) \\
\text { women }\end{array}$ & $\begin{array}{l}\text { APACHE II }{ }^{\mathrm{a}}(\text { mean } \pm \mathrm{SD} \text { or } \\
\left.\text { median }(\mathrm{IQR}) \text { or }(\mathrm{CI})^{*}\right)\end{array}$ \\
\hline \multirow{2}{*}{$\begin{array}{l}\text { Burtin et al. }{ }^{27)} \text {, } \\
2009\end{array}$} & I & 31 & $56 \pm 16$ & $9(29.03)$ & $26 \pm 6$ \\
\hline & $\mathrm{C}$ & 36 & $57 \pm 17$ & $10(27.8)$ & $25 \pm 4$ \\
\hline \multirow{2}{*}{$\begin{array}{l}\text { Schweickert et al.9), } \\
2009\end{array}$} & I & 49 & $57.7(36.3-69.1)$ & $29(59)$ & $20.0(15.8-24.0)$ \\
\hline & $\mathrm{C}$ & 55 & $54.4(46.5-66.4)$ & $23(42)$ & $19.0(13.3-23.0)$ \\
\hline \multirow{2}{*}{$\begin{array}{l}\text { Dantas et al. }{ }^{28)} \\
2012\end{array}$} & I & 14 & $59.07 \pm 15.22$ & $7(50)$ & $23.71 \pm 8.51$ \\
\hline & $\mathrm{C}$ & 14 & $50.43 \pm 20.45$ & $10(71.43)$ & $21.07 \pm 7.23$ \\
\hline \multirow{2}{*}{$\begin{array}{l}\text { Denehy et al. }{ }^{29)} \text {, } \\
2013\end{array}$} & I & 74 & $61.4 \pm 15.9$ & $31(41.9)$ & $19 \pm 6$ \\
\hline & $\mathrm{C}$ & 76 & $60.1 \pm 15.8$ & $24(31.6)$ & $20.7 \pm 7.7$ \\
\hline \multirow{2}{*}{$\begin{array}{l}\text { Collings\&Cusack }{ }^{30)} \text {, } \\
2015\end{array}$} & I: A & 5 & $61.4(44.68-78.12)^{*}$ & $1(20)$ & $16.8(15.04-26.16)^{*}$ \\
\hline & I: B & 5 & $59.2(31.43-86.97)^{*}$ & $3(60)$ & $20.6(12.86-20.74)^{*}$ \\
\hline \multirow{2}{*}{$\begin{array}{l}\text { Kayambu et al. }{ }^{31)} \text {, } \\
2015\end{array}$} & I & 26 & $62.5(30-83)$ & $8(16)$ & $28.0 \pm 7.6$ \\
\hline & $\mathrm{C}$ & 24 & $65.5(37-85)$ & $10(20)$ & $27.0 \pm 6.8$ \\
\hline \multirow{2}{*}{$\begin{array}{l}\text { Kho et al. }{ }^{32)} \\
2015\end{array}$} & I & 16 & $54 \pm 16$ & $9(56)$ & $25 \pm 8$ \\
\hline & $\mathrm{C}$ & 18 & $56 \pm 18$ & $8(50)$ & $25 \pm 6$ \\
\hline \multirow{2}{*}{$\begin{array}{l}\text { Moss et al. }{ }^{33)} \\
2015\end{array}$} & I & 59 & $56 \pm 14$ & $23(39)$ & $17.9 \pm 6.2$ \\
\hline & $\mathrm{C}$ & 61 & $49 \pm 15$ & $26(43)$ & $17.4 \pm 5.6$ \\
\hline \multirow{2}{*}{$\begin{array}{l}\text { Hodgson et al. }{ }^{34)} \\
2015\end{array}$} & I & 27 & $64 \pm 12$ & $8(38)$ & $19.8 \pm 9.8$ \\
\hline & $\mathrm{C}$ & 20 & $53 \pm 15$ & $12(41)$ & $15.9 \pm 6.9$ \\
\hline \multirow{2}{*}{$\begin{array}{l}\text { Fraser et al. }{ }^{35)} \text {, } \\
2015\end{array}$} & I & 66 & $65.8 \pm 19.6$ & $32(49)$ & $21.2 \pm 7.5$ \\
\hline & $\mathrm{C}$ & 66 & $63.5 \pm 14.6$ & $34(52)$ & $20.2 \pm 7.2$ \\
\hline \multirow{2}{*}{$\begin{array}{l}\text { Ota et al. }{ }^{36)}, \\
2015\end{array}$} & I & 48 & $64(46-73)$ & $14(31)$ & $14(11-20)$ \\
\hline & $\mathrm{C}$ & 60 & $72(59-82)$ & $16(27)$ & $16(12-21)$ \\
\hline
\end{tabular}

${ }^{a}$ APACHE II: Acute Physiological and chronic health evaluation II measures illness severity and is related to mortality risk; I: Intervention; C: Control; CI: Confidence Interval; IQR: Intercuartile Range.

The characteristics of the patients, interventions, measurements and results obtained were heterogeneous, and there were conflicted positions regarding the effectiveness of the interventions. Early rehabilitation was associated with an increase in functional capacity $\left.{ }^{9}, 35\right)$ and muscle strength ${ }^{27,28)}$, with a shorter duration of mechanical ventilation ${ }^{9}$, and an improvement in walking distance $\left.e^{9}, 27,32\right)$, as well as a better perception of the health-related quality of life $\mathrm{e}^{27}$.

The methodological evaluation has shown a lack of high quality studies. The most valued study was that by Kayambu et al. ${ }^{31)}$, although it did not reach the highest score due to the difficulty of the therapists being blinded. Other studies with medium-high methodological quality were the ones by Schweickert et al. ${ }^{9)}$ and Hodgson et al. ${ }^{34}$ ) in which, in addition, it was not possible that participants were blinded. In the rest of the research, the low quality (3-4 scores) is mainly explained by the lack of masking, intention to treat analysis, and by the significant differences of the individuals' characteristics, although these aspects have not been enough to consider their exclusion in the study.

In relation to the functional capacity measured through the Barthel scale, this review has found benefits, being significantly higher in the ICU intervention group at discharge from the Fraser et al. study ${ }^{35}$. This effect may be related to the significant presence of fewer patients undergoing mechanical ventilation in the intervention group.

In studies where electrostimulation ${ }^{31,32)}$ was applied, there was no significant increase in muscle strength at discharge, although successive measurements showed an improvement over the previous time interval ${ }^{32}$. In Kayambu et al. ${ }^{31}$, the positive effect of the intervention was associated with a systemic anti-inflammatory action. These results agree with a systematic review carried out in 2014, in which it was concluded that neuromuscular electrostimulation can be considered safe and effective to maintain the mass and muscle strength in the $\mathrm{ICU}^{37)}$ in such a way as to favour the prevention of ICUAW.

The results of this review show a significant increase in the distance walked at hospital discharge, 27,32 . However, patients in the study by Schweickert et al. ${ }^{9)}$ walked less meters than other patients from the rest of the studies. In the study by Kho et al. ${ }^{32)}$, the distance walked to the hospital was higher than the distance walked at discharge from the ICU. Likewise, in the study by Denehy et al. ${ }^{29}$, the data concerning the distance walked over the 3 months of follow-up was also higher. In parallel, the seconds used in the TUG test also decreased. In this regard, the probability of walking more meters can be explained by the use of more actively demanding interventions that focus on the musculature of the lower limbs such as the cycloergometer ${ }^{27,29)}$ or the neuromuscular electrostimulation ${ }^{32}$. Due to the lack of data on frequency, intensity, or duration, other considerations cannot be taken into account.

The perceived quality of life is related to the multifactorial morbidity suffered by patients who have survived a critical 


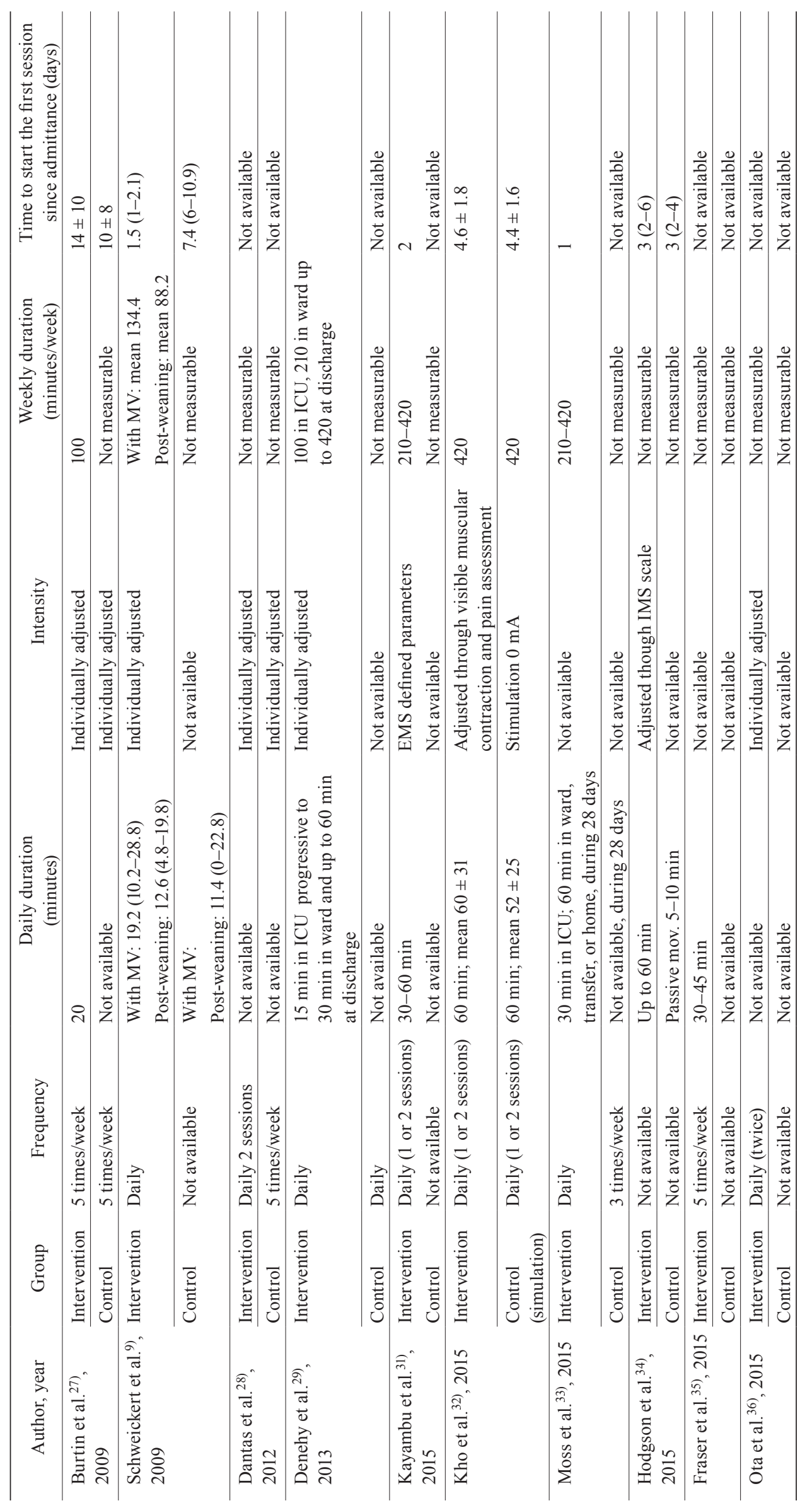


disease. This concept was valued only in three studies, so it is necessary to extend an integral conception of the patient that reflects the interest of highlighting the multidisciplinary role of the care team. The study by Burtin et al. ${ }^{27}$ ) showed a higher quality of life at discharge from the ICU, and Kayambu et al. ${ }^{31)}$ over the 6 months after discharge. These results confirm those assessed by the review by Kayambu et al. ${ }^{20)}$, where an improvement in the quality of life was also observed. However, this improvement was not only due to physical function, but also to cognitive aspects that were not valued.

It should be noted that the duration of mechanical ventilation was only less statistically significant in two studies ${ }^{9,36)}$. This result may be related to the daily interruption of sedation in the intervention group that could favour the weaning process. In any case, in the literature there is evidence of a shorter duration of mechanical ventilation associated with early mobilization $^{20,21)}$ that cannot be widely confirmed by this review.

In relation to the length of ICU or hospital stay, the results cannot confirm positive effects as opposed to other evidence from recent multicentre studies $^{38)}$.

The heterogeneity of the studies is also reflected in the variability of the care given to the control group, which includes activities of different types from rest to non-standardized mobilizations or mobilization protocols. In many cases, there is no exhaustive definition of the usual care given. Although early mobilization critical patients' care is considered important by professionals ${ }^{39)}$, these data suggest the presence of barriers and difficulties that need to be analysed in order to promote an environment of mobilization culture in the critical care units.

This review has some limitations. First of all, the systematic review strategy of search can have a potential selection bias due to the established criteria. As for the methodology of the included studies, the punctuation in the PEDro scale shows an absence of high quality studies. In most of the analysed investigations, changeability exists in the characteristics of the compared patients, so this must be taken into account when generalising the whole population of critical patients. Another limitation is the scarce number of studies found.

Future studies should include patients with similar illnesses and lines of clinical treatment. It is necessary to reflect on the measurable results and the instruments to be used. The dose, intensity, frequency, and duration of the interventions must be considered, as well as trying to obtain a detailed description both of the interventions and of the usual care given. It is also necessary to keep in mind the measurement of the quality of life, the rehabilitation sessions after discharge from the ICU, as well as the long-term effects.

As for conclusions, this review has included recent scientific studies on rehabilitation and early mobilization in ICU patients. In the analysed studies, a variety of interventions have been applied according to the existing literature.

The results have showed that rehabilitation and early mobilization produce an effect on the decrease of the days of admittance both at the ICU and at the hospital. On the contrary, we can affirm that there is an effect on the progress of the functional capacity, strength, mobility, quality of life, less duration of mechanical ventilation, and a higher probability of being discharged to home.

Rehabilitation and early mobilization are related to a higher probability of traveling more distance at discharge. In the interventions, the cycloergometer and muscular electro-stimulation can be used for the maintenance of the muscle strength.

In addition, an absence of continuity is observed in patients' follow-up after discharge from the ICU to ward, home, or in the long term. Therefore, it is necessary to make an effort to promote a culture of mobilization in the units of critical care.

The included studies have been heterogeneous and of few quality. Further research is necessary to establish more solid evidences on the effectiveness of the rehabilitation and early mobilization interventions.

\section{ACKNOWLEDGEMENT}

All authors have intellectually contributed to this work, meet all the authorship conditions and have approved the final version of the same.

Funding

Without funding.

Conflict of interest

None.

\section{REFERENCES}

1) Needham DM, Dennison CR, Dowdy DW, et al.: Study protocol: the Improving Care of Acute Lung Injury Patients (ICAP) study. Crit Care, 2006, 10: R9. [Medline] [CrossRef]

2) Engel HJ, Needham DM, Morris PE, et al.: ICU early mobilization: from recommendation to implementation at three medical centers. Crit Care Med, 2013, 41: S69-S80. [Medline] [CrossRef]

3) Herridge MS: Long-term outcomes after critical illness. Curr Opin Crit Care, 2002, 8: 331-336. [Medline] [CrossRef]

4) Cheung AM, Tansey CM, Tomlinson G, et al.: Two-year outcomes, health care use, and costs of survivors of acute respiratory distress syndrome. Am J Respir 
Crit Care Med, 2006, 174: 538-544. [Medline] [CrossRef]

5) Davydow DS, Katon WJ, Zatzick DF: Psychiatric morbidity and functional impairments in survivors of burns, traumatic injuries, and ICU stays for other critical illnesses: a review of the literature. Int Rev Psychiatry, 2009, 21: 531-538. [Medline] [CrossRef]

6) Fan E, Dowdy DW, Colantuoni E, et al.: Physical complications in acute lung injury survivors: a two-year longitudinal prospective study. Crit Care Med, 2014, 42: 849-859. [Medline] [CrossRef]

7) Cameron S, Ball I, Cepinskas G, et al.: Early mobilization in the critical care unit: a review of adult and pediatric literature. J Crit Care, 2015, 30: 664-672. [Medline] [CrossRef]

8) Hough CL, Needham DM: The role of future longitudinal studies in ICU survivors: understanding determinants and pathophysiology of weakness and neuromuscular dysfunction. Curr Opin Crit Care, 2007, 13: 489-496. [Medline] [CrossRef]

9) Schweickert WD, Pohlman MC, Pohlman AS, et al.: Early physical and occupational therapy in mechanically ventilated, critically ill patients: a randomised controlled trial. Lancet, 2009, 373: 1874-1882. [Medline] [CrossRef]

10) Hermans G, Van den Berghe G: Clinical review: intensive care unit acquired weakness. Crit Care, 2015, 19: 274. [Medline] [CrossRef]

11) Appleton RT, Kinsella J, Quasim T: The incidence of intensive care unit-acquired weakness syndromes: a systematic review. J Intensive Care Soc, 2015, 16: 126-136. [Medline] [CrossRef]

12) Farhan H, Moreno-Duarte I, Latronico N, et al.: Acquired muscle weakness in the surgical intensive care unit: nosology, epidemiology, diagnosis, and prevention. Anesthesiology, 2016, 124: 207-234. [Medline] [CrossRef]

13) Fan E: Critical illness neuromyopathy and the role of physical therapy and rehabilitation in critically ill patients. Respir Care, 2012, 57: 933-944, discussion 944-946. [Medline] [CrossRef]

14) Amidei C: Mobilisation in critical care: a concept analysis. Intensive Crit Care Nurs, 2012, 28: 73-81. [Medline] [CrossRef]

15) Stiller K: Physiotherapy in intensive care: an updated systematic review. Chest, 2013, 144: 825-847. [Medline] [CrossRef]

16) Castro-Avila AC, Serón P, Fan E, et al.: Effect of early rehabilitation during intensive care unit stay on functional status: systematic review and meta-analysis. PLoS One, 2015, 10: e0130722. [Medline] [CrossRef]

17) Bailey P, Thomsen GE, Spuhler VJ, et al.: Early activity is feasible and safe in respiratory failure patients. Crit Care Med, 2007, 35: 139-145. [Medline] [CrossRef]

18) Morris PE, Goad A, Thompson C, et al.: Early intensive care unit mobility therapy in the treatment of acute respiratory failure. Crit Care Med, 2008, 36: 2238-2243. [Medline] [CrossRef]

19) Adler J, Malone D: Early mobilization in the intensive care unit: a systematic review. Cardiopulm Phys Ther J, 2012, 23: 5-13. [Medline]

20) Kayambu G, Boots R, Paratz J: Physical therapy for the critically ill in the ICU: a systematic review and meta-analysis. Crit Care Med, 2013 , 41: 1543-1554. [Medline] [CrossRef]

21) Li Z, Peng X, Zhu B, et al.: Active mobilization for mechanically ventilated patients: a systematic review. Arch Phys Med Rehabil, 2013, 94: 551-561. [Medline] [CrossRef]

22) Hermans G, De Jonghe B, Bruyninckx F, et al.: Interventions for preventing critical illness polyneuropathy and critical illness myopathy. Cochrane Database Syst Rev, 2014, (1): CD006832. [Medline]

23) Calvo-Ayala E, Khan BA, Farber MO, et al.: Interventions to improve the physical function of ICU survivors: a systematic review. Chest, 2013, 144: 1469-1480. [Medline] [CrossRef]

24) Urrútia G, Bonfill X: Declaración PRISMA: una propuesta para mejorar la publicación de revisiones sistemáticas y metaanálisis. Med Clin (Barc) [Internet], 2010 135(11): 507-511. http://linkinghub.elsevier.com/retrieve/pii/S0025775310001454 (Accessed Jul. 17, 2017)

25) Maher CG, Sherrington C, Herbert RD, et al.: Reliability of the PEDro scale for rating quality of randomized controlled trials. Phys Ther, 2003, 83: 713-721. [Medline]

26) Cumming TB, Thrift AG, Collier JM, et al.: Very early mobilization after stroke fast-tracks return to walking: further results from the phase II AVERT randomized controlled trial. Stroke, 2011, 42: 153-158. [Medline] [CrossRef]

27) Burtin C, Clerckx B, Robbeets C, et al.: Early exercise in critically ill patients enhances short-term functional recovery. Crit Care Med, 2009, 37: 2499-2505. [Medline] [CrossRef]

28) Dantas CM, Silva PF, Siqueira FH, et al.: Influence of early mobilization on respiratory and peripheral muscle strength in critically ill patients. Rev Bras Ter Intensiva, 2012, 24: 173-178 (In Portuguese). [Medline] [CrossRef]

29) Denehy L, Skinner EH, Edbrooke L, et al.: Exercise rehabilitation for patients with critical illness: a randomized controlled trial with 12 months of follow-up. Crit Care, 2013, 17: R156. [Medline] [CrossRef]

30) Collings N, Cusack R: A repeated measures, randomised cross-over trial, comparing the acute exercise response between passive and active sitting in critically ill patients. BMC Anesthesiol, 2015, 15: 1. [Medline] [CrossRef]

31) Kayambu G, Boots R, Paratz J: Early physical rehabilitation in intensive care patients with sepsis syndromes: a pilot randomised controlled trial. Intensive Care Med, 2015, 41: 865-874. [Medline] [CrossRef]

32) Kho ME, Truong AD, Zanni JM, et al.: Neuromuscular electrical stimulation in mechanically ventilated patients: a randomized, sham-controlled pilot trial with blinded outcome assessment. J Crit Care, 2015, 30: 32-39. [Medline] [CrossRef]

33) Moss M, Nordon-Craft A, Malone D, et al.: A randomized trial of an intensive physical therapy program for patients with acute respiratory failure. Am J Respir Crit Care Med, 2016, 193: 1101-1110. [Medline] [CrossRef]

34) Hodgson CL, Bailey M, Bellomo R, et al. Trial of Early Activity and Mobilization Study Investigators: A binational multicenter pilot feasibility randomized controlled trial of early goal-directed mobilization in the ICU. Crit Care Med, 2016, 44: 1145-1152. [Medline] [CrossRef]

35) Fraser D, Spiva L, Forman W, et al.: Original research: implementation of an early mobility program in an ICU. Am J Nurs, 2015, 115: 49-58. [Medline] [CrossRef]

36) Ota H, Kawai H, Sato M, et al.: Effect of early mobilization on discharge disposition of mechanically ventilated patients. J Phys Ther Sci, 2015, 27: 859-864. [Medline] [CrossRef]

37) Wageck B, Nunes GS, Silva FL, et al.: Application and effects of neuromuscular electrical stimulation in critically ill patients: systematic review. Med Intensiva, 2014, 38: 444-454. [Medline] [CrossRef]

38) Wahab R, Yip NH, Chandra S, et al.: The implementation of an early rehabilitation program is associated with reduced length of stay: a multi-ICU study. J Intensive Care Soc, 2016, 17: 2-11. [Medline] [CrossRef]

39) Barber EA, Everard T, Holland AE, et al.: Barriers and facilitators to early mobilisation in intensive care: a qualitative study. Aust Crit Care, 2015, 28: 177-182, quiz 183. [Medline] [CrossRef] 